\title{
Entropy Changes in Rarefaction Waves ${ }^{1}$
}

\author{
Robert F. Dressler
}

\begin{abstract}
Frictional flow of a polytropic gas is investigated for centered rarefaction waves. Energy balance is maintained by assuming that mechanical energy loss due to frictional force reappears as heat. Entropy behavior at the wave front is discussed. The nonhomogeneous linear system of partial differential equations with variable coefficients for first-order frictional effects is derived. Utilizing the geometrical similarity of the Mach lines, the functional form of these quantities is ascertained, which permits explicit solutions of the boundary value problem. The expansion procedure is singular at the wave front, and results are not applicable there. First-order effects are expressed as polynomials plus terms singular at the wave front. Results are compared with expressions obtained when heat generation due to frictional force is neglected.
\end{abstract}

\section{Introduction}

Equations for one dimensional, unsteady flow of a compressible gas permit explicit solution for centered rarefaction waves, such as occur in shock tubes or pneumatic control circuits. For some applications, however, it becomes important to include also the complications resulting from frictional dissipation. This matter is discussed here in two ways, first with the simplifying assumption that the frictional effects consist merely in a retarding force, and second, by the more complete consideration that includes the resulting heat generation and accompanying change in entropy. This means that the mechanical energy destroyed by the retardation force reappears as heat energy, resulting in additional expansion. The simpler flow description without heat generation will be referred to as model $\mathrm{A}$, and the second, more accurate, one as model B. The equations for steady frictional flow in model $\mathrm{B}$ have been studied and yield energy theorems and an extended form of Bernoulli's theorem [1], ${ }^{2}$ but knowledge of the unsteady flows appears to be restricted mainly to a few isolated numerical calculations.

A centered rarefaction wave is perhaps the most basic pattern in unsteady flow; the present paper derives solutions for first-order dissipative effects in this unsteady wave using the full system of equations of model B, and compares results with corresponding ones for model A (which were previously published by the author [2] as a supplementary result in a study of shock tubes with varying cross section).

A recent paper by Ludford and Martin [3] has discussed anisentropic effects in centered simple waves. They consider flows where the specific entropy varies from particle to particle, but remains constant for a given particle. In frictional flow, however, the specific entropy for a given particle must vary with time, and a centered rarefaction wave cannot remain a simple wave.

Consider a polytropic gas at rest for time $\bar{t}<0$ in a duct of uniform cross section, filling the duct to the left of a diaphragm where $\bar{x}<0$, and with a vacuum initially at $\bar{x}>0$. Heat exchange between the gas and the duct, heat conduction and radiation, and viscous effects are disregarded. When the diaphragm is removed at $\bar{t}=0$, a centered rarefaction wave begins. Dimensional quantities are denoted by bars in order to define the unbarred dimensionless quantities to be used subsequently. Before flow, the gas state is described by sound speed $\bar{c}_{0}$, mass density $\bar{\rho}_{0}$, and temperature $\bar{T}_{0}$; after flow we have velocity $\bar{u}$, local sound speed $c$, mass density $\bar{\rho}$, and pressure $\bar{p}$.

For model A, assuming no internal heat generation and no entropy changes, the equation of state can be written in forms

$$
\bar{p}=\bar{A}^{*} \bar{\rho}^{\gamma}, \quad \bar{c}^{2}=\gamma \bar{A}^{*} \bar{\rho}^{\gamma-1}=\gamma \bar{R} \bar{T},
$$

1 This research was supported by the Office of Naval Research, USN.

2 Figures in brackets indicate the literature references at the end of this paper. 
where $\gamma$ is the adiabatic exponent, $\bar{R}$ the gas constant, $\bar{T}$ the temperature, and $\bar{A}^{*}$ is a known constant (consistent with the assumption here of no entropy change). In this simpler model, the friction produces merely a retarding force per unit mass of magnitude $\lambda \bar{u}^{2}$ where $\lambda$ is the known friction factor. There is some question concerning the constancy of this coefficient for frictional flows that are highly unsteady; this question is discussed by Schultz-Grunow [4], Jenny [5], and others. Here it is considered to be a constant.

Introducing dimensionless (unbarred) variables defined by $x=\bar{x} / \bar{D}, t=\left(\overline{c_{0}} / \bar{D}\right) \bar{t}, u=\bar{u} / \bar{c}_{0}$, $c=\bar{c} / \bar{c}_{0}, \rho=\bar{\rho} / \bar{\rho}_{0}, \lambda=\bar{D} \bar{\lambda}$ where $\bar{D}$ is some length associated with duct cross section, the momentum and continuity equations for model $\mathrm{A}$ are

$$
\left.\begin{array}{c}
u_{t}+u u_{x}+\frac{2}{\gamma-1} c c_{x}=-\lambda u|u| \\
c u_{x}+\frac{2}{\gamma-1} u c_{x}+\frac{2}{\gamma-1} c_{t}=0
\end{array}\right\}
$$

for the two unknowns $u(x, t)$ and $c(x, t)$; and the energy equation is neglected.

In deriving equations for model B one must take account of the mechanical energy lost through the action of the retarding force $\lambda u|u|$. The action of a body force cannot itself directly change entropy; however, if a term is included in the energy equation for an amount of generated heat equal to the lost mechanical energy, this heat will change the specific entropy. In this way, the energy equation becomes expressible directly in terms of the friction coefficient. The equation of state for model B now includes the entropy dependence,

$$
\bar{p}=\bar{A}(\bar{s}) \bar{\rho}^{\gamma}, \quad \bar{A}(\bar{s})=(\gamma-1) e^{\left(\bar{s}-\bar{s}_{\rho}\right) / c_{v}}, \quad \bar{c}^{2}=\gamma \bar{R} \bar{T}(\bar{\rho}, \bar{s})
$$

where $\bar{c}_{v}$ is specific heat at constant volume, $\bar{s}$ is the specific entropy (entropy per unit mass), and $\bar{s}_{o}$ is a constant with a value depending unon the entropy level adopted. Letting dimensionless specific entropy $s$ be defined by $s=\left(\bar{T}_{0} / \bar{c}_{0}{ }^{2}\right) \bar{s}$, the momentum, continuity, and energy equations for model B become, respectively,

$$
\left.\begin{array}{l}
u_{t}+u u_{x}+\frac{2}{\gamma-1} c c_{x}=-\lambda u|u|+c^{2} s_{x} \\
c u_{x}+\frac{2}{\gamma-1} u c_{x}+\frac{2}{\gamma-1} c_{t}=\lambda \gamma \frac{|u|^{3}}{c} \\
s_{t}+u s_{x}=\lambda \frac{|u|^{3}}{c^{2}}
\end{array}\right\}
$$

for the three unknowns $u(x, t), c(x, t)$, and $s(x, t)$. We set $\bar{s}=s=0$ for the gas when initially at rest, and the equality

determines the constant $\bar{s}_{0}$.

$$
\bar{A}^{*}=\bar{A}(0)=(\gamma-1) e^{-\bar{s}_{0} / \bar{c}_{\mathrm{o}}}
$$

\section{Characteristic Equations}

The Mach Jines for model B are defined by the system of characteristic equations equivalent to the totally hyperbolic system (4). These consist of three families of real curves with directions given by

$$
\left.\begin{array}{ll}
\zeta_{1}: & \frac{d x}{d t}=u-c \\
\zeta_{2}: & \frac{d x}{d t}=u+c \\
\zeta_{3}: & \frac{d x}{d t}=u
\end{array}\right\}
$$




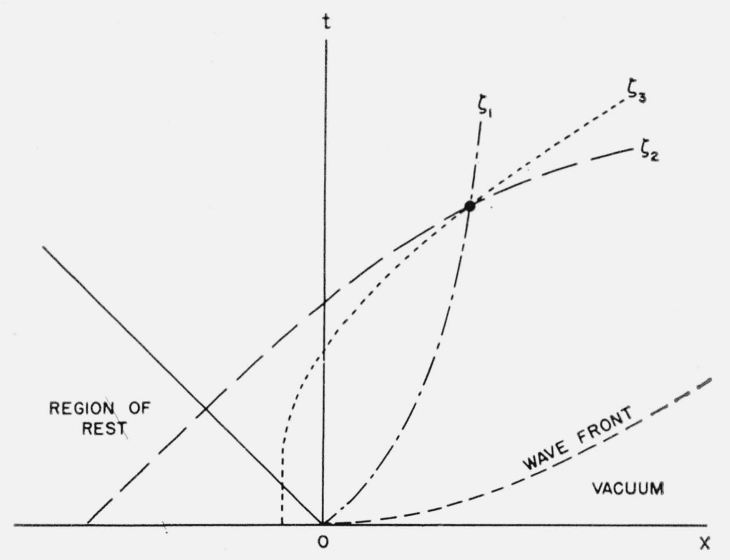

FIGURE 1 .

which are respectively the backward sound path, the forward sound path, and the particle trajectory (see fig. 1). The corresponding differential relations along each family are

$$
\left.\begin{array}{rlrl}
\zeta_{1}: & d\left(u-\frac{2}{\gamma-1} c\right)+c d s & =-\lambda u|u|\left(1+[\gamma-1] \frac{u}{c}\right) d t \\
\zeta_{2}: & d\left(u+\frac{2}{\gamma-1} c\right)-c d s & =-\lambda u|u|\left(1-[\gamma-1] \frac{u}{c}\right) d t \\
\zeta_{3}: & d s=\lambda \frac{|u|^{3}}{c^{2}} d t .
\end{array}\right\}
$$

These equations are used in [6] as the basis for a discussion of a numerical computation on a rarefaction wave in a shock tube.

\section{Entropy Behavior at the Wave Front}

From the above equations and the equation of state (3), the behavior of $s$ at the forward wave front where $\rho=0$ can be inferred, consistent with the assumed model for the flow. For notational simplicity and to permit numerical results, we now specialize the gas to be air with $\gamma=7 / 5$; however, the same arguments will apply and analogous solutions can be derived for any other admissible value of $\gamma$.

If $\bar{s}_{0}$ is chosen as indicated and the relation $\gamma=1+\bar{R} / \bar{c}_{v}$, is used, we obtain the dimensionless equation of state in the form $c^{2}=e^{\gamma(\gamma-1) s} \rho^{\gamma-1}$, which for air is

$$
\rho=e^{-7 s / 5} c^{5} .
$$

On the wave front curve where $\rho=0$, this permits two possibilities: either (a), $s$ remains finite and hence $c=0$ there, or (b), $s$ becomes infinite and $c$ is not known in advance. If $s$ were finite and continuous and $c$ vanished there, then by the third equation in (7), the rate of change of $s$ along the wave front would be everywhere infinite as $u \neq 0$ there. This follows from the fact that the wave-front locus is also a particle trajectory and therefore a $\zeta_{3}$ Mach line. Therefore $s$ must actually be infinite on the wave-front curve, and $c$ is not determined there by this argument. Because $s$ is entropy per mass, this does not necessarily imply that entropy per volume becomes infinite at the wave front for this model.

\section{First-Order Effects}

By setting up asymptotic expansions of form

$$
\left.\begin{array}{l}
v(x, t, \lambda) \sim v^{0}(x, t)+V^{A}(x, t) \lambda+\ldots . \\
v(x, t, \lambda) \sim v^{0}(x, t)+V^{B}(x, t) \lambda+\ldots(\operatorname{model} \mathrm{A}) \\
\text { (mol B })
\end{array}\right\}
$$


a perturbation scheme will be defined to give information for small values of $\lambda$. The quantities $v(x, t, \lambda)$ represent each unknown $u, c, s$, and their various first derivatives. The unperturbed centered rarefaction wave is the known simple-wave solution

$$
\left.\begin{array}{l}
u^{0}=5(1+x / t) \\
c^{0}=\frac{1}{6}(5-x / t) \\
s^{0}=0
\end{array}\right\}
$$

which when $\lambda=0$ satisfies system (2) for model A and system (4) for model B. The first-order terms for model A are $U^{\mathrm{A}}, C^{\mathrm{A}}$, (and $S^{\mathrm{A}}=0$ ) and have been published in [2]. Now we proceed to solve the more complicated problem for $U^{\mathrm{B}}, C^{\mathrm{B}}$, and $S^{\mathrm{B}}$ and to compare these with the previous results.

The expansion $s \sim 0+S^{\mathrm{B}}(x, t) \lambda+\ldots$. shows at once that the perturbation procedure for model $\mathrm{B}$ cannot be expected to retain validity in the neighborhood of the forward wave front, since $s(x, t, \lambda)=\infty$ for any $\lambda>0$ on the wave front whereas $s(x, t, 0)=0$ there. The results therefore will not apply in the neighborhood of the forward wave front where a type of boundary layer effect occurs. A separate analysis would be required in that region, possibly similar to the application of the Pohlhausen method as used by Whitham [7] for a water wave problem. There would be little justification for it in this problem, however, because the model assumed for frictional dissipation would in any case become inaccurate in this region of extremely low density. (A singular situation does not occur in the perturbation for model A. In that case there is no edge layer effect, and results hold throughout the full wave; but on the other hand, the basic model itself is everywhere less accurate than model B.)

When expansions of type (9) are inserted into system (4), after using (10), the first-order terms satisfy the system:

$$
\left.\begin{array}{l}
\frac{(5 t-x)^{2}}{t^{2}} S_{x}^{\mathrm{B}}-\frac{30(t+x)}{t} U_{x}^{\mathrm{B}}-36 U_{t}^{\mathrm{B}}-\frac{30(5 t-x)}{t} C_{x}^{\mathrm{B}}-\frac{30}{t} U^{\mathrm{B}}+\frac{30}{t} C^{\mathrm{B}}+\frac{25(t+x)^{2}}{t^{2}}=0 \\
\frac{(5 t-x)}{t} U_{x}^{\mathrm{B}}+\frac{25(t+x)}{t} C_{x}^{\mathrm{B}}+30 C_{t}^{\mathrm{B}}-\frac{5}{t} U^{\mathrm{B}}+\frac{5}{t} C^{\mathrm{B}}-\frac{175(t+x)^{3}}{6 t^{2}(5 t-x)}=0 \\
\frac{5(t+x)}{t} S_{x}^{\mathrm{B}}+6 S_{t}^{\mathrm{B}}-\frac{125(t+x)^{3}}{t(5 t-x)^{2}}=0 .
\end{array}\right\}
$$

The solution of these equations that is sought must satisfy the conditions $U^{\mathrm{B}}=C^{\mathrm{B}}=S^{\mathrm{B}}=0$ on the straight line $t=-x,(t \geq 0)$. This nonhomogeneous linear system has the same characteristic curves as the solution $u^{0}, c^{0}$ plus its particle trajectories. By studying these curves one can infer an easy procedure for obtaining the desired functions. These curves in the flow wedge are

$$
\left.\begin{array}{ll}
\zeta_{1}^{0}: \quad x=m t, \quad-1 \leq m \leq 5 & \\
\zeta_{2}^{0}: \quad\left(\frac{x}{a}\right)=5\left(\frac{t}{a}\right)-6\left(\frac{t}{a}\right)^{2 / 3}, & 0<a \\
\zeta_{3}^{0}: \quad\left(\frac{x}{a}\right)=5\left(\frac{t}{a}\right)-6\left(\frac{t}{a}\right)^{5 / 6}, & 0<a
\end{array}\right\}
$$

where each equation defines a one-parameter family of curves over the range of a parameter as indicated. The $\zeta_{2}^{0}$ and $\zeta_{3}^{0}$ curves emanate from the back wave line $x=-t$ at a point $(-a, a)$ for any $a>0$ (see fig. 2). Writing $\zeta_{1}^{0}$ as $(x / a)=m(t / a)$, one sees that the coordinates of an intersection point of a fixed $\zeta_{\mathrm{I}}^{0}$ curve with any $\zeta_{2}^{0}$ curve must be proportional to the parameter $a$. Therefore as the parameter $a$ is varied, the quantities $t / a$ and $x / a$ at such intersection points remain constant as we progress along a fixed $\zeta_{1}^{0}$ ray. Likewise an analogous situation will 


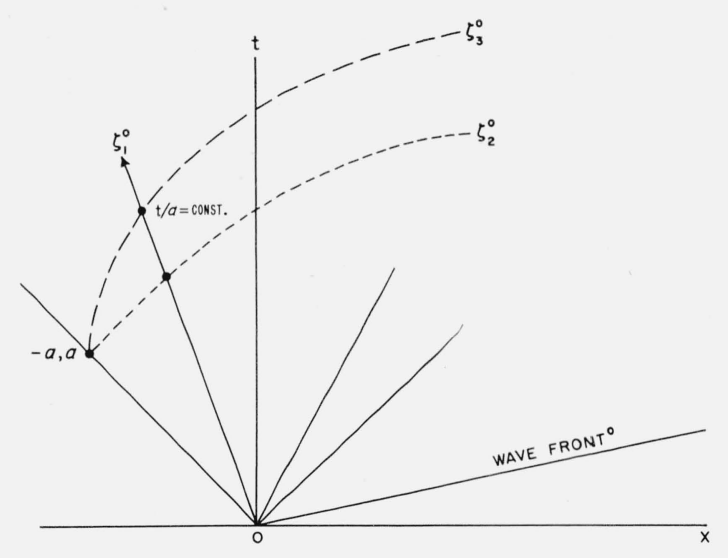

Figure 2.

apply for intersections of $\zeta_{3}^{0}$ curves with a fixed $\zeta_{1}^{0}$ ray. This geometric similarity of the characteristics can be employed to find the functional form of the desired solutions $U^{\mathrm{B}}, C^{\mathrm{B}}, S^{\mathrm{B}}$. The characteristic relations associated with (12) are

$$
\left.\begin{array}{l}
\zeta_{1}^{0}: \quad d\left(U^{\mathrm{B}}-5 C^{\mathrm{B}}\right)+\left(\frac{5 t-x}{6 t}\right) d S^{\mathrm{B}}=\left(\frac{25(x+t)^{3}}{18 t^{2}(x-5 t)}-\frac{25(x+t)^{2}}{36 t^{2}}\right) d t-\frac{5}{3} \frac{U^{\mathrm{B}}-C^{\mathrm{B}}}{t} d t \\
\zeta_{2}^{0}: \quad d\left(U^{\mathrm{B}}+5 C^{\mathrm{B}}\right)-\left(\frac{5 t-x}{6 t}\right) d S^{\mathrm{B}}=-\left(\frac{25(x+t)^{3}}{18 t^{2}(x-5 t)}+\frac{25(x+t)^{2}}{36 t^{2}}\right) d t \\
\zeta_{3}^{0}: \quad d S^{\mathrm{B}}=\frac{125(t+x)^{3}}{6 t(5 t-x)^{2}} d t .
\end{array}\right\}
$$

The last equation does not contain unknowns on the right side and could therefore be integrated along any $\zeta_{3}^{0}$ curve. If this were done, using the third equation in (12) and the geometric similarity, it is easily seen that it would lead to a relation of the form $S^{\mathrm{B}} / a=F_{3}(t / a)$ when $S^{\mathrm{B}}=0$ at the origin. Because $t / a$ is constant along $a \zeta_{1}^{0}$ ray, $S^{\mathrm{B}}$ is proportional to $a$, and hence to $t$ there; the functional form must then be $S^{\mathrm{B}}(x, t)=L(m) t$. Using this result for $d S^{\mathrm{B}}$ in the second equation of (13), by analogous argument applied to the $\zeta_{2}^{0}$ curves, it follows that $U^{\mathrm{B}}+$ $5 C^{\mathrm{B}}=F_{2}(m) t$. Finally, we see that if we integrate the first equation of (13) along any $\zeta_{1}^{0}$ ray (where $m=$ constant), we would have $\left(U^{\mathrm{B}}-5 C^{\mathrm{B}}\right)$ proportional to $t$ if the quantity $5\left(U^{\mathrm{B}}-C^{\mathrm{B}}\right) / 3 t$ were a constant along the ray. Then it would follow that $U^{\mathrm{B}}$ and $C^{\mathrm{B}}$ would separately be proportional to $t$ also. But in that case, $5\left(U^{\mathrm{B}}-C^{\mathrm{B}}\right) / 3 t$ would actually be constant as desired. There these considerations indicate the existence of a solution to (13) and (11), vanishing at the origin as required, with the functional form $f(m) t$ for each unknown $U^{\mathrm{B}}, C^{\mathrm{B}}$, and $S^{\mathrm{B}}$. This information now permits immediate solution of the original system (11), as follows:

First, system. (11) is transformed through use of the new independent variables $m$ and $t$, putting $U^{\mathrm{B}}(x, t)=U^{*}(m, t)$, and likewise for the other unknowns. Then setting $U^{*}=h(m) t$, $C^{*}=k(m) t$, and $S^{*}=L(m) t$ leads immediately to a nonhomogeneous system of three ordinary differential equations for $h, k$, and $L$. Although this system contains variable coefficients, it can nevertheless be solved exactly. The particular solution satisfying the conditions $h(-1)=$ $k(-1)=L(-1)=0$ is then finally obtained to give the first-order effects for model $\mathrm{B}$.

$$
\left.\begin{array}{l}
\frac{U^{\mathrm{B}}}{t}=\frac{25}{48}\left[\frac{35}{108}(5-m)^{3}-7(5-m)^{2}+54(5-m)-172+\frac{180}{(5-m)}\right] \\
\frac{C^{\mathrm{B}}}{t}=\frac{5}{48}\left[\frac{(5-m)^{7}}{72,576}+\frac{35}{54}(5-m)^{3}-15(5-m)^{2}+108(5-m)+(1+m)^{2}-\frac{1868}{7}+\frac{90}{(5-m)}+\frac{7(1+m)^{3}}{(5-m)}\right] \\
\frac{S^{\mathrm{B}}}{t}=125\left[\frac{(5-m)^{6}}{2,177,280}-\frac{1}{5}(5-m)+3-\frac{108}{7(5-m)}+\frac{27}{(5-m)^{2}}\right]
\end{array}\right\}
$$



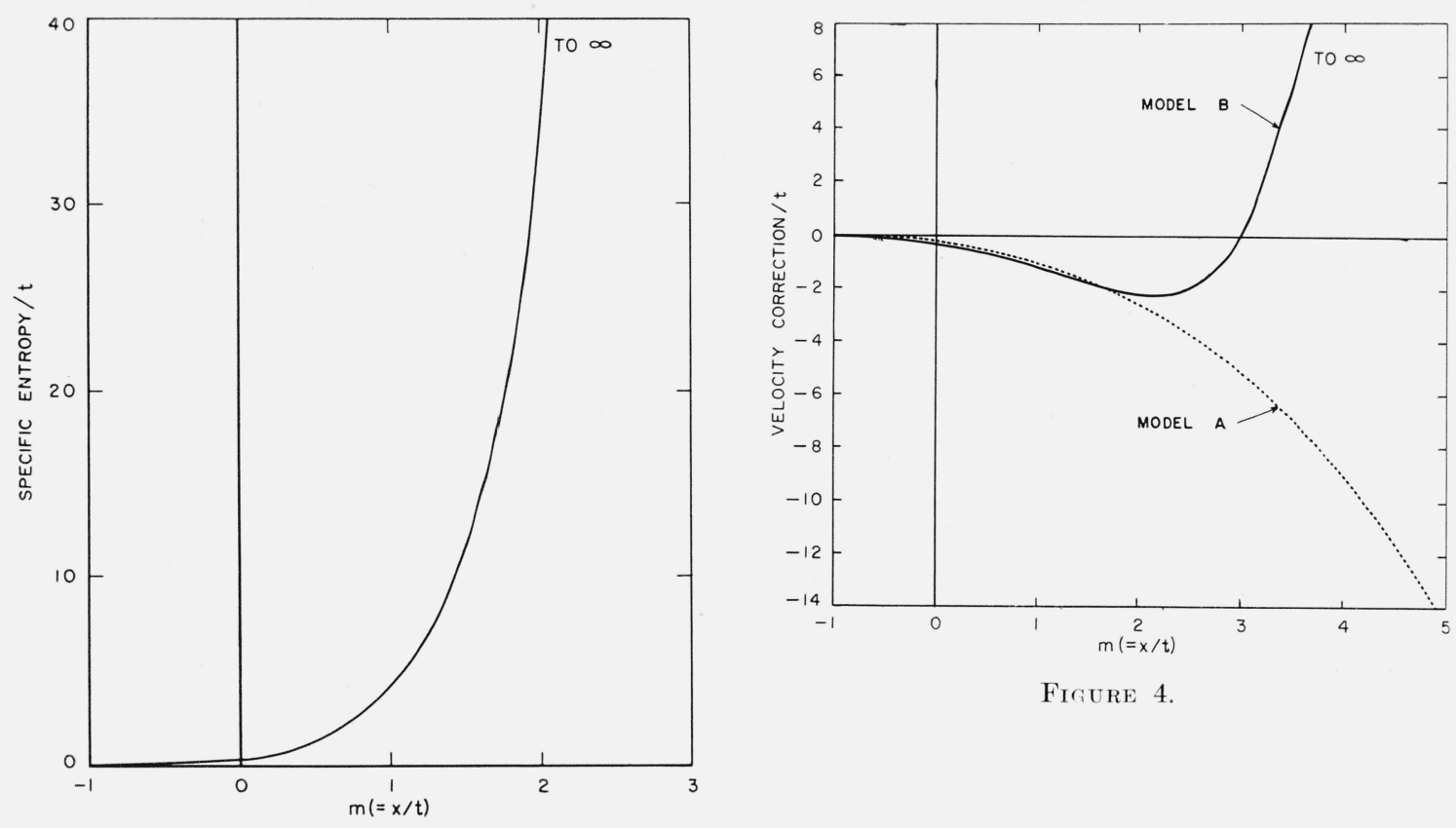

Figure 4.

FIFURE 3.

For comparison of these results with the corresponding expressions for the simpler flow model, we list below the expressions obtained in [2] for model A:

$$
\left.\begin{array}{l}
\frac{U^{\mathrm{A}}}{t}=\frac{25}{144}\left[\frac{2}{9}(5-m)^{3}-5(5-m)^{2}+36(5-m)-84\right] \\
\left.\frac{C^{\mathrm{A}}}{t}=\frac{5}{144}\left[\frac{4}{9}(5-m)^{3}-10(5-m)^{2}+72(5-m)-168+3(m+1)^{2}\right]\right\} \\
\frac{S^{\mathrm{A}}}{t}=0 .
\end{array}\right\}
$$

The expressions in (14) have singularities at $m=5$, at the forward wave front for the unperturbed flow. This is to be expected in view of the previous remarks concerning the entropy behavior at the forward wave front that makes the perturbation procedure singular. On the other hand, the expansion for model A does not possess this singular property, and the expressions (15) do not become infinite at $m=5$. A comparison of (14) with (15) must be restricted to a region that excludes the forward wave-front zone. Figure 3 gives the numerical evaluation of the quantity $S^{\mathrm{B}} / t$. When drawn on a semilogarithmic plot, this curve is almost linear over the middle range $1<m<4$, and for convenience can be accurately approximated there by the expression $.63 \exp (1.95 \mathrm{~m})$. Figure 4 compares the behavior of $U^{\mathrm{B}} / t$ with $U^{\mathrm{A}} / t$. In the first half of the flow zone, the two models predict almost identical results, but further on the velocity becomes higher for model B. In equations (4), as $s_{x}>0$ for this case, the effect of the entropy term in the momentum equation is to diminish the total resistive force, and this by itself would increase the velocity. In the continuity equation, however, the extra positive term on the right side would have the effect by itself of decreasing the velocity in the back portion of the flow zone, because this term is analogous to the term that would be present for a converging duct in quasi-one-dimensional flow, as derived in [2]. Evidently the fact that the velocity term for model B is slightly below the model A curve in the beginning of the flow zone, is due to the extra term in the continuity equation dominating over the entropy term in the momentum equation until $s_{x}$ grows sufficiently large to raise the velocity. 

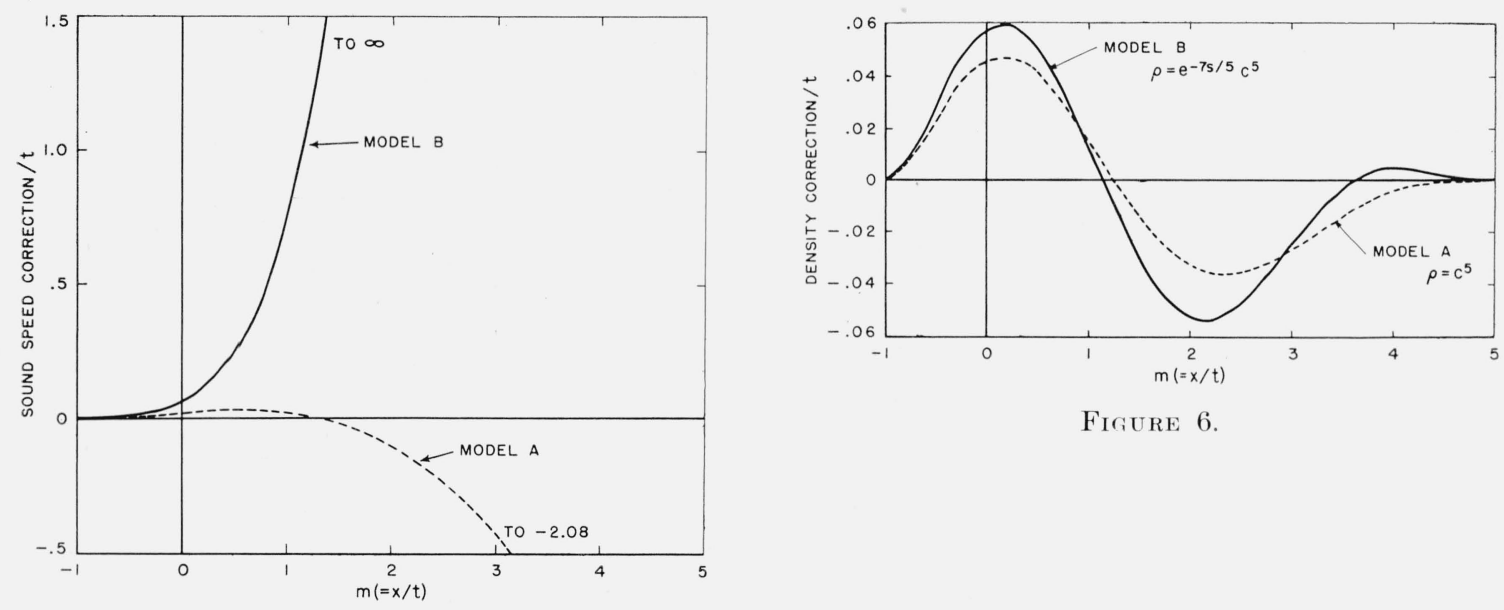

Figure 6.

Figure 5.

The first-order corrections for the local sound speed $c$ are shown in figure 5 for the two models. In this case there is no resemblance in the behavior of the two curves, as the sound speed, which is proportional to the square root of the temperature, is more directly sensitive than the velocity to the internal heat generation assumed in model B.

Finally we use our results for sound speed and entropy in the two models to calculate the corrections for the dimensionless density $\rho=\bar{\rho} / \bar{\rho}_{0}$. The dimensionless equation of state of air for model A corresponding to (1) is $\rho=c^{5}$. After inserting expansions here, the coefficient of $\lambda$ in the expansion of type (9) for $\rho$ is then equal to $5\left(c^{0}\right)^{4} C^{\mathrm{A}}$. This quantity, after division by $t$, is shown in figure 6. For model B, when corresponding expansions are inserted in (8), the coefficient of $\lambda$ in the $\rho$ expansion is equal to $5\left(c^{0}\right)^{4}\left[C^{\mathrm{B}}-\left(7 c^{0} S^{\mathrm{B}} / 25\right)\right]$. This quantity, after division by $t$, is likewise shown in figure 6 , and one observes that the widely different results of the two models for sound speed and entropy nevertheless combine to produce density corrections that are in close agreement. The pressure and temperature behavior for the wave can be obtained directly from (3) using the results already presented.

\section{References}

[1] L. Prandtl, Fluid dynamies (Blackie \& Sons, Ltd., London, 1952).

[2] R. F. Dressler, Turbulent flow in shock tubes of varying cross section, J. Research NBS 53, 253 (1954) RP2541.

[3] G. S. S. Ludford and M. H. Martin, One-dimensional anisentropic flows, Comm. Pure Appl. Math. VII, No. 1 (New York University, Feb. 1954).

[4] F. Schultz-Grunow, Pulsierender Durchfluss durch Rohre, Forschg. Ing. Wes. 11 (1940).

[5] E. Jenny, Berechnungen und Modellversuche über Druckwellen grosser Amplituden in Auspuff-Leitungen, Zürich, E.T.H., Promotionsarbeit, Basel (1949).

[6] N. A. Hall, The action of friction in non-steady flow of fluids (First Midwestern Conf. on Fluid Dynamics, Edwards, Ann Arbor, Mich., 1951).

[7] G. B. Whitham, Effects of hydraulic resistance in the dam-break problem, Proc. Roy. Soc. London [A] 227 (Jan. 1955).

Washington, March 6, 1956. 